3. University of Colorado Anschutz Medical Campus, Aurora/CO/ United States of America

4. Emergency Medicine, University of Colorado Anschutz Medical Campus, Aurora/CO/United States of America

Study/Objective: To determine community level awareness of risk factors for stroke and cardiovascular disease, in a remote and medically underserved region of Ghana.

Background: Hypertension and other non-communicable diseases are growing risk factors for cardiovascular disease and stroke in developing countries. A multi-region survey from a central clinic investigating participants' level of awareness and education surrounding hypertension and stroke, provides important information to guide primary prevention and public health response.

Methods: A central clinic in Nkonya-Wurupong, Ghana, evaluated 1,671 patients in July 2016, and a group of 302 adults over the age of 18 provided a convenience sampling. The survey examined three main areas; demographics, medical history and knowledge deficit with respect to stroke and cardiovascular risk factors.

Results: Fifty-six participants demonstrated hypertension (BP $>139 / 89$ ), of which 17 were male and 37 female. One-hundred and six believed hypertension was a risk factor for stroke. Twenty-six were medicated for hypertension. The majority of the participants believed that modifiable factors put them at risk for stroke, and that stroke was preventable. Diet, heart disease, smoking, obesity, diabetes, sedentary lifestyle or alcohol were not identified as risk factors. One-sided weakness was consistently associated with stroke. Other symptoms included in the survey were headache, slurred speech, visual changes, dizziness, and facial droop. It was difficult to discern the sources of participants' information. A few respondents did indicate school, internet, radio, TV, medical books, or health professionals.

Conclusion: Knowledge of the link between hypertension, cardiovascular disease and stroke varies significantly, along with stroke-symptom identification and sources of medical information. Many participants indicated the belief that stroke can be prevented, however it is unclear what respondents believe modifiable risk factors consist of. This data suggests there are major areas where healthcare education is needed. Discerning baseline health and medical knowledge in remote and developing regions, is essential for disaster preparedness and primary prevention.

Prehosp Disaster Med 2017;32(Suppl. 1):s208-s209

doi:10.1017/S1049023X17005441

\section{Telemedicine Consultations in an All-Russian Center}

\section{Disaster Medicine}

Gennady Kipor ${ }^{1}$, Igor Shilkin ${ }^{2}$, Vladimir Petlah ${ }^{3}$, Vladimir

Rosinov ${ }^{3}$, Andrew Kilnik ${ }^{2}$, Vladimir Shilkin ${ }^{2}$, Sergei

Goncharov $^{4}$

1. International Department, All Russian Center for Disaster medicine, Moscow/Russian Federation

2. Department Of Information, All Russian Center for Disaster medicine, Moscow/Russian Federation

3. International Department, Pirogov Russian National Research Medical University, Moscow/Russian Federation

4. Director Of Ardmc, All Russian Center for disaster Medicine "Zaschita", Moscow/Russian Federation
Study/Objective: Analyze telemedicine consultations made in the All-Russian Center for Disaster Medicine (ARCDM).

Background: The territory of the country is more than 11 billion square miles, and there is no sufficient, medically qualified staff centers.

Methods: The structure of the provision of telemedicine consultations (TMC) of Russian disaster medicine service includes center of control crisis situations, having a connection with 21 federal hospitals and regional centers of disaster medicine, and having contact with the republican (regional) hospitals. ARCDM have mobile telemedicine complexes, based mobile satellite communication VSAT-stations, for use in emergency situations, which provides a system to quickly deploy remote support operations for rescuers and medical staff of field hospitals.

Results: Analysis of 115 TMCs was performed during the 2015 in ARCDM. Requests for telemedicine consultations came from different regions of the country. Leading experts of the federal medical centers in Moscow conducted TMCs. The most frequent requests were for neurosurgery profile - $26.9 \%$, intensive care $(21.7 \%$ traumatology, $14.7 \%$ neurology, $10.5 \%$ pediatrics, $5 \%$ cardiovascular surgery and oncology), and other $6.1 \%$. As a result of TMCs, correction of medical care was made in $53.2 \%$ of patients, an accurate diagnosis and treatment plan was $32.2 \%$, and $15.6 \%$ of patients were evacuated for treatment in specialized centers. The mobile telemedicine complex has been included in equipment of field hospitals, working at Northern Caucasus. In total, 121 telemedicine consultations were performed during 1.5 months (33.3\% to children). Ten patients were delivered directly for further treatment to central hospitals; nine patients after TMC diagnostics were specified with a treatment plan in field hospitals.

Conclusion: We note the high efficiency use of TMC for an establishment of the diagnosis and medical tactics and operative decision of questions of evacuation of patients. Keywords: telemedicine, emergency situations, and mobile telemedicine complex.

Prehosp Disaster Med 2017;32(Suppl. 1):s209

doi:10.1017/S1049023X17005453

\section{Evaluating Aviation Accidents in the World from 2003} to 2016

Ebru Inal ${ }^{1}$, Edip Kaya ${ }^{2}$

1. Civil Defense and Firefighting, Yalova University, Yalova/Turkey

2. Public Health, Hacettepe University, Ankara/Turkey

Study/Objective: This study aims to determine some features of aviation accidents and to examine existing statistics on aviation accidents over the past 10 years worldwide.

Background: It is important to mitigate losses due to aviation accidents through aviation accident prevention measures in the disaster management cycle.

Methods: Data was obtained from the records of planecrashinfo.com (an accident database). The data included dates, times, flight number, aircraft type, total aboard (passengers/ crew), total fatalities aboard (passengers/crew) locations of accidents and intent of flights. In this descriptive study, 
statistical analysis was carried out for features of each incident. The Chi-square test was applied to compare the fatalities and types of flights (domestic or international flights). Grouping of numbers of fatalities and survivors was done according to previous studies.

Results: The data reported a total of 619 aviation accidents which occurred within 13 -year period. The number of accidents decreased from 2003 to 2016 with the exception of 2008. There were 12,339 lives lost due to aviation accidents which occurred between the study period. While $64.8 \%(n=397)$ of accidents had no survivors only $2.0 \%(\mathrm{n}=12)$ of accidents had no fatality. Passenger flights (49.1\%) constituted a greater proportion of the accidents. Thirty-one out of 33 accidents resulted in 100 + fatalities, and 18 out of 22 accidents resulted in 50 to 99 fatalities. Aviation accidents occurred in 115 different countries. The number of fatalities which were $100+$ and above was significantly higher in international flights $(53.1 \%)$ as compared to the domestic flights $(46.9 \%)(\mathrm{p}<0.001)$.

Conclusion: Aviation accidents and fatalities related to these accidents were high between 2003 and 2016 years worldwide. Passenger flights were responsible for the majority of fatalities and accidents. More detailed studies and interventions are needed to reduce the rate of aviation accidents.

Prehosp Disaster Med 2017;32(Suppl. 1):s209-s210

doi:10.1017/S1049023X17005465

An Evaluation of Records Related to Dwelling Fires in the Central District of Yalova Province, Turkey

Ebru Inal ${ }^{1}$,Edip Kaya ${ }^{2}$, Bahar G. Dogan ${ }^{2}$

1. Civil Defence and Firefighting, Yalova University, Yalova/Turkey

2. Public Health, Hacettepe University, Ankara/Turkey

Study/Objective: This analysis aims to evaluate the dwelling fires in Central District of Yalova Province between the years from 2010 to 2015.

Background: Fires are one of the most common disasters and important cause of accidental death in world and also in Turkey. It is fourth leading cause of death within unintentional injuries with a proportion of $4 \%$.

Methods: A total of 3014 fires were recorded by Yalova Provincial Fire Authority within the study period. Out of them 630 dwelling fires (\%20.9) were evaluated. In this descriptive study, permission was obtained from the Provincial Fire Department to analyze the data. The data consisted of the cause, type and year of fire, degree of burning. Losses caused by fire were not recorded.

Results: Chimney ignition was the first cause of dwelling fires with percent of $35.2 \%(\mathrm{n}=222)$ followed by electricity $(33.0 \%$, $\mathrm{n}=208)$ and smokers' materials $(2.4 \%, \mathrm{n}=15) .94 .6 \%$ $(n=596)$ of fires was extinguished by firefighters at the beginning phase. The distribution of the fires by year has not shown a steady increase: $19.0 \%(\mathrm{n}=120)$ was happened in 2010, $18.5 \%(\mathrm{n}=116)$ in $2011,15.5 \%(\mathrm{n}=98)$ in 2012 , $16.2 \%(\mathrm{n}=101)$ in $2013,13.5 \%(\mathrm{n}=85)$ in $2014,17.3 \%$ $(n=109)$ in 2015. 99.3\% $(n=626)$ of the construction material was concrete and $0.7 \%(n=4)$ was wooden for all dwelling fires. It was interesting that the losses (human and/or animal) were not recorded.
Conclusion: The number of dwelling fires consisted of one-fifth of total fires and the number was not decreased by years. All dwelling fires should be regarded as potentially preventable events, effective and intensive public training activities should be carried on the reasons of fires and prevention.

Prehosp Disaster Med 2017;32(Suppl. 1):s210

doi:10.1017/S1049023X17005477

Was Internet Usage Effective on Radiation Protection After the Nuclear Disasters among General Workers in

Fukushima?

Hideyuki Kanda ${ }^{1}$, Kenzo Takahashi ${ }^{2}$, Nagisa Sugaya ${ }^{3}$,Hideki

Tsumura ${ }^{1}$, Mari Fukuda ${ }^{1}$, Kikuo Koyama

1. Shimane University, Izumo/Japan

2. Teikyo University, Tokyo/Japan

3. Yokohama City University, Yokohama/Japan

4. Fukushima Occupational Health Promotion Center, Fukushima/ Japan

Study/Objective: To clarify radiation knowledge and preventive behaviors between those who got the information on the Fukushima Dai-ichi Nuclear Power Plants' Accidents (FDNPPAs) mainly by the Internet, and those who didn't among general workers in Fukushima, 3-5 months after a nuclear disaster.

Background: The FDNPPAs were the second nuclear power plant accidents in the world. On the other hand, there is a widespread diffusion of the Internet all over the world. No studies have been done regarding the relationship between using the Internet and radiation knowledge, and preventive behaviors after a nuclear disaster.

Methods: A descriptive study of preventive behaviors among general workers in Fukushima, 3 to 5 months following the nuclear accidents. The subjects were 1,394 regular workers who took part in health seminars produced by the Fukushima Occupational Health Promotion Center between July and September, 2011. Of the 1,282 responses, 1,119 eligible responses participated in this study. This anonymous questionnaire survey was asking for characteristics and questions on main information sources following the nuclear accidents. Those who chose the Internet on main information sources were defined as Internet users. We also asked 10 questions of radiation knowledge (hair loss, cancer, malformation etc.) and 10 questions of preventive behaviors (washing hands, wearing a mask, refraining from going outside etc.).

Results: Workers who got the information on the FDNPPAs mainly by the Internet, had more radiation knowledge among general workers in Fukushima after a nuclear disaster. Plus, the Internet users had more preventive behaviors against radiation than non-users.

Conclusion: The Internet was one of useful tools for having knowledge and preventive behaviors in emergency settings such as nuclear disasters. Our research will contribute to determine a way of distributing information to average citizens if a nuclear accident occurs in the future.

Prehosp Disaster Med 2017;32(Suppl. 1):s210

doi:10.1017/S1049023X17005489 